\title{
Chronic silica gel poisoning - two year follow up
}

\author{
Kamal Abdul Naser ${ }^{1}$, Dhakshila Niyangoda ${ }^{2}$ \\ Journal of the Ceylon College of Physicians, 2013, 44, 38-39
}

\begin{abstract}
A 43-year-old woman with a history of hyperthyroidism presented to the hospital with recurrent heart burn, muscular pain, difficulty in walking, giddiness and numbness in all four limbs. She had a history of hyperthyroidism and had been on carbimazole $20 \mathrm{mg}$ twice daily for 6 months and ingested beads of silica gel provided as a desiccant in bottles with carbimazole tablets. She was treated with rabeprazole $20 \mathrm{mg}$ daily and carbimazole $10 \mathrm{mg}$ daily while she was being investigated. After completion of investigations, she was reassured and carbimazole was continued. She was followed up at six months, twelve months and two years. She was symptom free. We believe this is the first reported case of chronic ingestion of silica gel.
\end{abstract}

\section{Case report}

A 43-year-old woman with a history of hyperthyroidism presented to the hospital with recurrent heart burn, muscular pain, difficulty walking, giddiness and numbness in all four limbs. She had a history of hyperthyroidism and had been on carbimazole $20 \mathrm{mg}$ twice daily for 6 months. She had been issued carbimazole in bottles, which contain silica gel in small packs. As she had been instructed to take four tablets twice a day, she had started taking four tablets of carbimazole and four beads of silica gel twice a day. When the dose was reduced, she had reduced the dose of silica gel accordingly.

After about three months of use, she presented to the doctor with muscle pain, numbness of all four limbs and unsteadiness on walking. However, her symptoms were ignored and she was reassured since clinical examination was normal. After six months on a routine inspection of left over medicines, it was found that she was taking silica gel two pellets twice a day with her carbimazole tablets. She was referred to the

\footnotetext{
${ }^{1}$ Teaching Hospital, Peradeniya, Sri Lanka.

2 Department of Pharmacy, Faculty of Allied Health Sciences, University of Peradeniya, Peradeniya, Sri Lanka.
}

toxicology unit for further investigations and management.

On clinical examination, she was euthyroid. Nervous and all the other systems were clinically normal. Laboratory investigations revealed normal full blood count, erythrocyte sedimentation rate (ESR), renal function, liver profile and normal urine analysis. Electrocardiogram (ECG), chest xray and ultrasonography of abdomen were normal. Magnetic resonance imaging (MRI) scan of brain and posterior fossa, which was done in the view of unsteadiness on walking and nerve conduction studies of peripheral nerves, did not reveal any abnormalities. Upper gastro-intestinal endoscopy, which was done because of recurrent heart burn, proved to be normal.

She was treated with rabeprazole $20 \mathrm{mg}$ daily and carbimazole $10 \mathrm{mg}$ daily. Her heart burn disappeared with rabeprazole. After completion of investigations, she was reassured and carbimazole was continued. She was followed up for six months and was symptom free. We followed up her six months, twelve months and twenty four months. We could not find any clinical or biochemical abnormalities up to twenty four months.

\section{Discussion}

Patient's symptoms were most probably related to the ingestion of silica gel. However the symptoms could have been attributed to side effects of carbimazole because side effects of carbimazole include nausea, mild gastro-intestinal disturbances, headache, rashes, pruritus and arthralgia ${ }^{1}$. However the patient was relieved when ingestion of silica gel was discontinued.

Silica gel is a partially dehydrated polymeric form of colloidal silicic acid with the formula $\mathrm{SiO}_{2} \cdot \mathrm{nH}_{2} \mathrm{O}$. This amorphous material is composed of spherical particles, 2-20 nm in size, which aggregate to form the adsorbent, with pore sizes in the range of $6-25 \mathrm{~nm}$. Surface areas are in the range of $100-850 \mathrm{~m}^{2} / \mathrm{g}$, depending on whether the gel is low density or regular density. The surface comprises mainly $\mathrm{SiOH}$ and SiOSi groups and, being polar, it can be used to adsorb water, alcohols, phenols, amines etc. (i.e. polar compounds) by hydrogen bonding mechanisms ${ }^{2}$. Therefore, this patient's symptoms can be attributed to these properties. 
Silica gel is used for drying procedures in which high capacity is required at low temperature and moderate water vapour pressure ${ }^{2}$. Therefore, it is used as a desiccant and is included with medicines, which deteriorate in the presence of moisture. According to Bough, when pets ingest silica gel granules or packets, there is little risk for serious side effects. Gastrointestinal upset may occur, especially in sensitive pets, but symptoms usually are easily managed at home without veterinary intervention ${ }^{3}$. Riordan et al. have categorized silica gel as a low toxic substance on ingestion for children ${ }^{4}$. However, there are no reported cases of chronic exposure to silica gel and we believe this is the first reported case of long term ingestion of silica gel.

Silica gel can cause a drying irritation of the mucous membranes and skin in cases of severe exposure due to extensive absorption of water. Another possible harmful effect is that beads can shatter inside the digestive system. Consequently, they will act as tiny pieces of broken glass inside the gut and damage the gut wall. These effects may have led to recurrent heart burn, which was successfully treated with rabeprazole.
This case report illustrates that chronic ingestion of silica gel does not seem to cause significant acute or chronic problems in humans. The silica gel sachet that is found with many medicines and food items caries a warning that it should not be swallowed. However it is only written in English in small print. Most Sri Lankans would not be able to read English. Therefore it is vital that any important information is given in a language that could be read and understood by the native population of that country. The font size of the letters also matters when this type of information is given to patients.

\section{References}

1. bnf.org. British National Formulary 55. BMJ group and RPS publishing 2008; 377.

2. Crittenden B, Thomas WJ. Adsorption technology and design. Reed educational and professional publishing Ltd 1998.

3. Bough MG. Not quite nontoxic. Veterinary Technician 2004; 2.

4. Riordan M, Rylance G, Berry K. Poisoning in children 4: Household products, plants, and mushrooms. (N/A) (N/A);406. [online]Available. 